\title{
Recent developments in bacterial conjugate vaccines
}

\author{
D. GOLDBLATT
}

Immunobiology Unit, Institute of Child Health and Great Ormond Street Hospital for Children, 30 Guildford Street, London WCIN 1 EH

\begin{abstract}
Study of the epidemiology of childhood infection reveals that the brunt of disease for a number of invasive bacterial infections is borne by children under the age of 4 years. Haemophilus influenzae type b (Hib), Neisseria meningitidis and Streptococcus pneumoniae, the three most important causes of childhood meningitis, illustrate this phenomenon, which is caused by the inability of infants and young children to mount antibodies to the carbohydrates that form a capsule surrounding these organisms. Carbohydrates are traditionally viewed as $T$-independent antigens with a number of unique and important immunological properties that are not encountered when inducing an immune response to proteins. These properties include no overt requirement for the presence of $T$ cells to induce an immune response, dominance of $\operatorname{IgM}$, failure to induce memory following immunisation, an absence of affinity maturation following immunisation, and poor immunogenicity in infants, the elderly and the immunocompromised. These properties of carbohydrates have precluded the use of pure carbohydrate vaccines in those patients most at risk. Conjugate vaccine technology, where a carbohydrate antigen is coupled chemically to a protein carrier, has overcome the limitations of carbohydrates as vaccine antigens by rendering the carbohydrate moiety of such vaccines immunogenic, even in the very young. The dramatic success of the Hib conjugate vaccines, the first conjugates licensed clinically for human use, in reducing the incidence of invasive Hib disease has demonstrated the potential value of such conjugate vaccines. Similar technology is, therefore, being applied to a number of other vaccines in development, including $N$. meningitidis (groups $\mathrm{A}$ and $\mathrm{C}$ ) and $S$. pneumoniae vaccines. The large number of pneumococcal carbohydrate serotypes that require inclusion in a vaccine makes this conjugate formulation far more complicated than that for Hib, and it is likely that the dramatic success of the Hib conjugate vaccines will be more difficult to repeat for the pneumococcus.
\end{abstract}

\section{Introduction}

Neisseria meningitidis, Streptococcus pneumoniae and Haemophilus influenzae type b (Hib) are the three most important causes of bacterial meningitis after the neonatal period. These organisms have in common a carbohydrate capsule (although of different structure) that acts as both a virulence determinant and a target for protective antibody. The importance of these pathogens in the young, and particularly the peak incidence of bacterial meningitis in those aged $<2$ years, can partly be explained by the poor immuno-

Received 18 Dec. 1997; accepted 22 Jan. 1998.

Corresponding author: Dr D. Goldblatt. genicity of carbohydrates in this age group and the consequent inability of infants and young children to mount protective antibody responses to these organisms. Therefore, vaccines containing purified capsular carbohydrates alone have failed to protect this high risk group from infection. However, the recent success of the Hib glyco-conjugate vaccines in reducing the incidence of invasive Hib disease has paved the way for exciting developments in the field of meningococcal and pneumococcal conjugate vaccines.

\section{The immunology of conjugate vaccines}

Goebel and Avery first demonstrated in 1929 that the poor immunogenicity of purified $S$. pneumoniae type 3 
polysaccharide in rabbits could be enhanced by conjugation of the polysaccharide to a protein carrier [1]. At the basis of this observation lies the difference in the immune system's handling of the two basic classes of antigen. Naturally occurring carbohydrate (CHO) antigens tend to be large molecules consisting of repeating epitopes, and these are not processed by antigen-presenting cells (APCs), but interact directly with B cells, inducing antibody synthesis in the absence of $\mathrm{T}$ cells (thus designated T-independent antigens). $T$ cells can influence the antibody response to certain carbohydrates, such as the capsular polysaccharide of $S$. pneumoniae type 3 [2], but an absolute requirement for $\mathrm{T}$ cells has not been demonstrated.

As a consequence of the unique interaction of $\mathrm{CHOs}$ and the immune system, memory cells are not formed and, classically, no affinity maturation is seen. Furthermore, the response to CHOs is age restricted and poorly developed in those aged $<2$ years. In older children and adults, antibody responses are induced by $\mathrm{CHO}$ antigens, but they are dominated by $\operatorname{IgM}$ and are relatively short-lived. In adults, the IgG response tends to be IgG2 restricted, although IgG1 responses are also seen. In children, IgG1 responses dominate the immune response to CHOs and only later in childhood do IgG2 responses become apparent. When the immune system encounters exogenous protein antigen, the antigen is taken up by APCs such as monocytes, tissue macrophages or B cells. The proteins are then degraded and the resulting peptides complex with major histocompatibility complex (MHC) class II molecules and are re-expressed at the cell surface. $\mathrm{T}$ cells recognise these antigen:MHC complexes, in addition to binding to a number of accessory molecules expressed on the surface of APCs. Activated T cells then secrete various cytokines that orchestrate the subsequent immune response, which may involve antibody production or cellmediated immunity. The immune response to protein antigen is thus designated T-dependent because of the absolute requirement for $\mathrm{T}$ cell help. This response is not age-restricted and can be induced in young infants. Antibody responses to protein antigens tend to be dominated by the IgG1 and IgG3 subclasses and longterm memory is established for the relevant antigen. Furthermore, affinity maturation of the antibody specific for the protein antigen increases with time, presumably due to cognate interaction between antigen-specific B cells, the APC and T cells.

The early experiments of Goebel and Avery demonstrated that it was possible to change the immunological nature of a $\mathrm{CHO}$ antigen from that of a $\mathrm{T}$ independent to a T-dependent molecule by conjugating the $\mathrm{CHO}$ to a protein carrier. Their experiments led indirectly to the development of conjugate vaccines for use in man, of which the Hib conjugate vaccines have been the highly successful prototype.

\section{Vaccines to prevent Hib infection}

In addition to meningitis, invasive Hib causes a number of other infection syndromes in young children, including bacteraemia, pneumonia and epiglottitis. Vaccination to prevent Hib infection was first attempted with purified polyribosyl-ribitol phosphate (PRP), the CHO found in the capsule of type $b$ organisms. Initial studies showed the vaccine to be immunogenic in children and adults, but poorly immunogenic in infants aged $<18$ months [3], and the vaccine was shown not to induce memory [4]. Following animal studies, initial human infant studies confirmed the immunogenicity of PRP-conjugate vaccines $[5,6]$. Formulations with various protein carriers, including tetanus and diphtheria toxoids, mutant diphtheria toxin and outer-membrane protein (OMP), have been developed and have been shown to influence immunogenicity [7]. For example, the vaccine containing PRP conjugated to the OMPs of $N$. meningitidis has been shown to be immunogenic following a single dose in infancy, whereas the PRP-tetanus toxoid and diphtheria conjugate vaccines have demonstrated significant immunogenicity only after two, but more strikingly after three doses. In addition to being immunogenic in the very young, the conjugate vaccines have been shown to prime for memory responses. This has been demonstrated by comparing antibody responses to pure PRP at the age of 12-18 months in infants who did or did not receive PRP-conjugate vaccines in the first year of life [8]. Some researchers have used the rapidity with which antibody responses are mounted to a dose of pure PRP as a marker of memory [9], while others have explored the increase in antibody avidity over time as a surrogate marker of the successful generation of memory [10]. Interestingly, even the least immunogenic of the conjugates, PRP conjugated to diphtheria toxoid, has been shown to be effective in reducing the incidence of invasive Hib infection in Finland [11]. The efficacy of such formulations may be related to the ability of the conjugate vaccines to induce memory, even in the face of poor primary immunogenicity [8].

IgG subclass responses to the Hib conjugate vaccines in infancy have been demonstrated to be predominantly IgG1, and boosting with pure PRP generates a response that is dominated by this subclass, although IgG2 responses are also seen [12]. Carrier protein priming at the age of 1 month enhances the immune response to subsequent conjugate vaccines, which enables conjugates utilising diphtheria or tetanus protein to be interchanged during primary vaccine schedules that include diphtheria and tetanus combinations [13]. In contrast, carrier protein priming of newborn infants may be associated with subsequent suppression of the response to the Hib conjugates [14], while maternally acquired anti-carrier antibodies may inhibit the immune response of an infant to the conjugate vaccines [15]. 
Widespread use of the Hib conjugate vaccines as part of the routine infant immunisation schedule has demonstrated them to be highly effective in protecting infants from invasive Hib disease in many countries, including the USA, Finland and the UK $[11,16,17]$. The success of the Hib conjugate vaccines in reducing the incidence of disease in vaccinated populations is caused partly by the reduction of the nasopharyngeal carriage of $\mathrm{Hib}$ in the vaccinees, and the resulting interruption in the person-to-person spread of the organism. Of particular importance has been a recent study of Hib conjugate vaccines in the Gambia [18], where administration of the vaccine together with diphtheria, tetanus, pertussis (DTP) vaccine and polio vaccine at the age of 6,10 and 14 weeks resulted in a striking reduction in invasive $\mathrm{Hib}$ disease, as well as a $25 \%$ overall reduction in the incidence of pneumonia. While the epidemiology of invasive Hib disease in the developing world has not received enough attention, this report provides optimism that the new generation conjugates, if made affordable, could make a real impact on mortality and morbidity in the under-five age group worldwide.

However, the complexity of the immunology of the new generation conjugate vaccines has been revealed by Eskola and colleagues [19], who demonstrated the reduced immunogenicity of Hib conjugate vaccines when given mixed with DTP vaccines in which the pertussis component was acellular. It would appear that the adjuvant aluminium hydroxide, included in most DTP combinations, interferes with the immunogenicity of the DT-acellular pertussis-PRP combinations, resulting in reduced Hib responses. Changing the aluminium salt to aluminium phosphate appears to abolish this effect [20]. Future combination vaccines must be developed and evaluated with caution. The successful development of Hib conjugates has provided optimism for the similar development of effective meningococcal and pneumococcal vaccines.

\section{The prevention of $N$. meningitidis and S. pneumoniae infections}

Conjugate vaccines specific for $N$. meningitidis and $S$. pneumoniae are currently under development and at various stages of clinical evaluation. Three major capsular types of $N$. meningitidis, A, B and C, cause most clinical invasive meningococcal disease worldwide. While group A disease is confined largely to epidemic disease in sub-Saharan Africa and the developing world, disease caused by groups $\mathrm{B}$ and $\mathrm{C}$ accounts for most infection in the developed world. In the UK, $32 \%$ of invasive $N$. meningitidis reports received by the Public Health Laboratory Meningococcal Reference Unit in 1996 were associated with group $\mathrm{C}$ isolates. Groups $\mathrm{A}$ and $\mathrm{C}$ have carbohydrate capsules that, as described previously, are virulence factors and targets for bactericidal antibody. Most efforts at conjugation have therefore focused on these two groups, and a recent study from the Gambia has confirmed the immunogenicity of the meningococcal group A plus group $\mathrm{C}$ mutant diphtheria toxin conjugate in infants [21]. Studies of a meningococcal $\mathrm{A}+\mathrm{C}$ conjugate vaccine administered as part of the UK infant immunisation schedule at the age of 2, 3 and 4 months have shown the vaccine to be well tolerated and immunogenic [22]. However, antibody titres and bactericidal activity fall rapidly following primary immunisation; hence it is possible that a booster dose may be required at the age of 1 year. Ultimately, the prevention of meningococcal disease worldwide will require the availability of a safe and effective serogroup $B$ vaccine. The development of a group $B$ vaccine is hampered by the non-immunogenicity of the B capsule, probably as a result of the immunogenic tolerance that arises out of the similarity between the capsular polysaccharide structure and the polysialic acid-containing glycopeptides that are components of human brain tissue. While alternative antigens being evaluated currently include OMPs of the dominant subtypes that cause infection, some researchers have sought crossreactive carbohydrate epitopes from other bacterial species. One such vaccine contains the chemically modified $N$-propionylated polysialic acid from Escherichia coli strain $\mathrm{K} 1$ capsule conjugated to purified recombinant meningococcal porin, and this has been shown recently to elicit bactericidal anti-serogroup B polysaccharide antibodies in baboons and rhesus monkeys [23]. There was relatively little cross-reaction with unmodified $\mathrm{N}$-acetyl polysaccharide in both mice and primates.

The development of conjugate pneumococcal vaccines remains an important goal because of the worldwide importance of $S$. pneumoniae as a pathogen in infants, the immunocompromised and the elderly. In the light of increasing $S$. pneumoniae antibiotic resistance, both in the UK and abroad [24-26], the need for an effective, universally administrable pneumococcal vaccine is becoming urgent. Current formulations of the pneumococcal vaccine contain a mixture of the purified capsular polysaccharide derived from 23 different types of $S$. pneumoniae. Despite 25 years of clinical use, the efficacy of these unconjugated vaccines in at-risk populations remains controversial $[27,28]$, while the limitations of their use in young infants has been outlined above. Current strategies for the conjugation of pneumococcal polysaccharides are following the lines of those demonstrated already to be effective for Hib, although the challenge of including purified capsular carbohydrates from as many clinically relevant serotypes as possible makes the design of the vaccine different to that for Hib. As a result of physical limitations, it is likely that conjugated pneumococcal vaccines will contain a maximum of 11 serotypes, and the choice of serotypes for inclusion in the vaccine is therefore crucial. The most relevant serotypes for inclusion in a vaccine will 
vary according to a number of factors, including the target age group, the type of pneumococcal infection to be prevented and the area of the world for which the vaccine is intended [29-32]. Early phase II studies in human infants have shown the vaccine to be immunogenic [33-35] and to prime for memory responses [36], although differences between the serotypes have been noted [37]. Encouragingly, the vaccine has also been shown to be immunogenic in high risk children with HIV infection [38], and efficacy studies in high risk groups are eagerly awaited.

One of the important mechanisms by which the Hib conjugate vaccines have reduced the incidence of invasive disease is by the reduction of nasopharyngeal carriage of Hib. Studies of nasopharyngeal carriage of $S$. pneumoniae have thus far been limited in their follow-up time, although the potential for the increase in nasopharyngeal carriage of pneumococcal serotypes not included in the vaccine has already been noted [39-41]. The danger that such increased carriage may lead to invasive disease, replacing the disease prevented by the serotypes contained in the vaccine, is real and needs to be evaluated carefully in studies with sufficient power to detect such an increase.

\section{Conclusions}

Conjugate vaccine technology, as applied to Hib vaccines, has proved highly successful in preventing invasive Hib infections. The approach used successfully for the design, manufacture and implementation of Hib conjugate vaccine is now being applied to the development of vaccines against other important encapsulated pathogens. The large number of pneumococcal polysaccharide antigens that require inclusion in a single vaccine and the unique nature of the group B meningococcal polysaccharide are but two of the problems facing conjugate vaccine development and implementation, and suggest that the success of the Hib vaccines may not be as simple to repeat. The vision of an infant vaccine schedule that incorporates antigens from all the major childhood pathogens, including $N$. meningitidis, Hib and $S$. pneumoniae, is still some way off. Indeed, the sheer volume of antigen, and in particular of conjugate protein, suggests that a multiple-antigen 'meningitis' vaccine given as a single injection will still require much development.

\section{References}

1. Avery OT, Goebel WF. Chemo-immunological studies on conjugated carbohydrate-proteins. II. Immunological specificity of synthetic sugar-protein antigens. $J$ Exp Med 1929; 50: 533550.

2. Baker PJ. $T$ cell regulation of the antibody response to bacterial polysaccharide antigens: an examination of some general characteristics and their implications. $J$ Infect Dis 1992; 165 Suppl 1: S44-48.

3. Peltola H, Käyhty H, Sivonen A, Mäkelä H. Haemophilus influenzae type $\mathrm{b}$ capsular polysaccharide vaccine in children: $\mathrm{a}$ double-blind field study of 100,000 vaccinees 3 months to 5 years of age in Finland. Pediatrics 1977; 60: 730-737.

4. Käyhty H, Karanko V, Peltola H, Mäkelä PH. Serum antibodies after vaccination with Haemophilus influenzae type b capsular polysaccharide and responses to reimmunization: no evidence of immunologic tolerance or memory. Pediatrics 1984; 74: $857-865$.

5. Zahradnik JM, Gordon LK. Augmented antibody [Ab] responses in infants administered a new Haemophilus influenzae type b capsular polysaccharide [PRP] diphtheria toxoid conjugate vaccine [PRP-D]. Pediatr Res 1984; 18: 289 A.

6. Ward J, Berkowitz C, Pescetti J, Burkart K, Samuelson J, Gordon L. Enhanced immunogenicity in young infants of a new Haemophilus influenzae type b (HIB) capsular polysaccharide (PRP)-diphtheria toxoid (D) conjugate vaccine Pediatr Res 1984; 18: 287A.

7. Granoff DM, Anderson EL, Osterholm MT et al. Differences in the immunogenicity of three Haemophilus influenzae type b conjugate vaccines in infants. J Pediatr 1992; 121: 187-194.

8. Käyhty H, Eskola J, Peltola H, Saarinen L, Mäkelä PH. High antibody responses to booster doses of either Haemophilus influenzae capsular polysaccharides or conjugate vaccine after primary immunization with conjugate vaccines. $J$ Infect Dis 1992; 165 Suppl 1: S165-166.

9. Zepp F, Schmitt HJ, Kaufhold A et al. Evidence for induction of polysaccharide specific B-cell-memory in the 1st year of life: plain Haemophilus influenzae type b-PRP (Hib) boosters children primed with a tetanus-conjugate Hib-DTPa-HBV combined vaccine. Eur J Pediatr 1997; 156: 18-24.

10. Goldblatt D, Pinto Vas R, Miller E. Antibody avidity as a surrogate marker of successful priming following Haemophilus influenzae type b conjugate vaccine. J Infect Dis 1997 (in press).

11. Peltola H, Kilpi T, Anttila M. Rapid disappearance of Haemophilus influenzae type $\mathrm{b}$ meningitis after routine childhood immunisation with conjugate vaccines. Lancet $1992 ; 340$ : 592-594.

12. Ambrosino DM, Sood S, Lee MC et al. IgG1, IgG2 and IgM responses to two Haemophilus influenzae type b conjugate vaccines in infants. Pediatr Infect Dis $J$ 1992; 11: 855-859.

13. Goldblatt D, Fairley CK, Cartwright K, Miller E. Interchangeability of conjugated Haemophilus influenzae type $\mathrm{b}$ vaccines during the primary immunisation of infants. $B M J 1996 ; 312$ : $817-818$.

14. Lieberman JM, Greenberg DP, Wong VK et al. Effect of neonatal immunization with diphtheria and tetanus toxoids on antibody responses to Haemophilus influenzae type b conjugate vaccines. $J$ Pediatr 1995; 126: 198-205.

15. Barington T, Gyhrs A, Kristensen K, Heilmann C. Opposite effects of actively and passively acquired immunity to the carrier on responses of human infants to a Haemophilus influenzae type b conjugate vaccine. Infect Immun 1994; 62: $9-14$.

16. Santosham M, Wolff M, Reid R et al. The efficacy in Navajo infants of conjugate vaccine consisting of Haemophilus influenza type b polysaccharide and Neisseria meningitidis outer-membrane protein complex. $N$ Engl J Med 1991; 324: 1767-1772.

17. Booy R, Heath PT, Slack MPE, Begg N, Moxon ER. Vaccine failures after primary immunisation with Haemophilus influenzae type-b conjugate vaccine without booster. Lancet 1997; 349: 1197-1202.

18. Mulholland K, Hilton S, Adegbola R et al. Randomised trial of Haemophilus influenzae type-b tetanus protein conjugate for prevention of pneumonia and meningitis in Gambian infants. Lancet 1997; 349: 1191-1197.

19. Eskola J, Ölander R, Hovi T, Litmanen L, Peltola S, Käyhty H Randomised trial of the effect of co-administration with acellular pertussis DTP vaccine on immunogenicity of Haemophilus influenzae type $\mathrm{b}$ conjugate vaccine. Lancet 1996; 348: $1688-1692$

20. Mills EL, Russell M, Cunning L et al. A fully liquid acellular pertussis vaccine combined with IPV and Hib vaccines (DTaPIPV-PRP-T) is safe and immunogenic without significant interaction. ICAAC 1997; G-95 [Abstract]. 
21. Twumasi PA, Kumah S, Leach A et al. A trial of a group A plus group $\mathrm{C}$ meningococcal polysaccharide-protein conjugate vaccine in African infants. $J$ Infect Dis 1995; 171: 632-638.

22. Fairley CK, Begg N, Borrow R, Fox AJ, Jones DM, Cartwright $\mathrm{K}$. Conjugate meningococcal serogroup $\mathrm{A}$ and $\mathrm{C}$ vaccine: reactogenicity and immunogenicity in United Kingdom infants. $J$ Infect Dis 1996; 174: 1360-1363.

23. Fusco PC, Michon F, Tai JY, Blake MS. Preclinical evaluation of a novel group $\mathrm{B}$ meningococcal conjugate vaccine that elicits bactericidal activity in both mice and nonhuman primates. J Infect Dis 1997; 175: 364-372.

24. George RC, Ball LC, Cooper PG. Antibiotic-resistant pneumococci in the United Kingdom. Commun Dis Rep CDR Rev 1992; 2: R37-43.

25. Simberkoff MS. Drug-resistant pneumococcal infections in the United States. A problem for clinicians, laboratories, and public health. JAMA 1994; 271: 1875-1876.

26. Privitera G. Penicillin resistance among Streptococcus pneumoniae in Europe. Diagn Microbiol Infect Dis 1994; 19: 157161.

27. Simberkoff MS, Cross AP, Al-Ibrahim M et al. Efficacy of pneumococcal vaccine in high-risk patients: results of a Veterans Administration cooperative study. $N$ Engl $J \mathrm{Med}$ 1986; 315: 1318-1327.

28. Shapiro ED, Berg AT, Austrian R et al. The protective efficacy of polyvalent pneumococcal polysaccharide vaccine. $N$ Engl J Med 1991; 325: 1453-1460.

29. Scott JAG, Hall AJ, Dagan $\mathrm{R}$ et al. Serogroup-specific epidemiology of Streptococcus pneumoniae: associations with age, sex, and geography in 7,000 episodes of invasive disease. Clin Infect Dis 1996; 22: 973-981.

30. Sniadack DH, Schwartz B, Lipman $\mathrm{H}$ et al. Potential interventions for the prevention of childhood pneumonia: geographic and temporal differences in serotype and serogroup distribution of sterile site pneumococcal isolates from children - implications for vaccine strategies. Pediatr Infect Dis J 1995; 14: $503-510$.

31. Greenwood BM, Hassan-King M, Macfarlane JT et al. Pneumococcal serotypes in West Africa. Lancet 1980; 1: 360.
32. Mastro TD, Ghafoor A, Nomani NK et al. Antimicrobial resistance of pneumococci in children with acute lower respiratory tract infection in Pakistan. Lancet 1991; 337: $156-159$.

33. Leach A, Ceesay SJ, Banya WAS, Greenwood BM. Pilot trial of a pentavalent pneumococcal polysaccharide/protein conjugate vaccine in Gambian infants. Pediatr Infect Dis $J$ 1996; 15: $333-339$.

34. Åhman H, Käyhty H, Tamminen P, Vuorela A, Malinoski F, Eskola J. Pentavalent pneumococcal oligosaccharide conjugate vaccine PncCRM is well tolerated and able to induce an antibody response in infants. Pediatr Infect Dis $J$ 1996; 15: 134-139.

35. Pichichero ME, Shelly MA, Treanor JJ. Evaluation of a pentavalent conjugated pneumococcal vaccine in toddlers. Pediatr Infect Dis $J$ 1997; 16: 72-74.

36. Anderson EL, Kennedy DJ, Geldmacher KM, Donnelly J, Mendelman PM. Immunogenicity of heptavalent pneumococcal conjugate vaccine in infants. $J$ Pediatr 1996; 128: 649-653.

37. O'Brien KL, Steinhoff MC, Edwards K, Keyserling H, Thoms ML, Madore D. Immunologic priming of young children by pneumococcal glycoprotein conjugate, but not polysaccharide, vaccines. Pediatr Infect Dis $J$ 1996; 15: 425-430.

38. King JC, Vink PE, Farley JJ et al. Comparison of the safety and immunogenicity of a pneumococcal conjugate with a licensed polysaccharide vaccine in human immunodeficiency virus and non-human immunodeficiency virus-infected children. Pediatr Infect Dis J 1996; 15: 192-196.

39. Dagan R, Melamed R, Muallem $\mathrm{M}$ et al. Reduction of nasopharyngeal carriage of pneumococci during the second year of life by a heptavalent conjugate pneumococcal vaccine. $J$ Infect Dis 1996; 174: 1271-1278.

40. Obaro SK, Adegbola RA, Banya WAS, Greenwood BM. Carriage of pneumococci after pneumococcal vaccination. Lancet 1996; 348: 271-272.

41. Mbelle N, Wasas A, Huebner R, Kimura A, Chang I, Klugman $\mathrm{K}$. Immunogenicity and impact on carriage of 9-valent pneumococcal conjugate vaccine given to infants in Soweto, South Africa. ICAAC 1997; LB21 [Abstract]. 